\title{
CXCR3, CXCR5, CXCR6, and CXCR7 in Diabetes
}

Poupak Fallahi ${ }^{1}$, Alda Corrado ${ }^{1}$, Andrea Di Domenicantonio ${ }^{1}$, Giada Frenzilli ${ }^{1}$, Alessandro Antonelli ${ }^{1}$, Silvia Martina Ferrari ${ }^{1}$.

${ }^{1}$ Department of Clinical and Experimental Medicine, University of Pisa, Via Savi, 10, I-56126 Pisa, Italy.

\begin{abstract}
Many studies have suggested that CXCR3, CXCR5, CXCR6 and CXCR7 chemokine receptors play a crtical role in the autoimmune process and in $\beta$-cell destruction in type 1 diabetes (T1D). In particular circulating CXCL10 level (the ligand of CXCR3) is high in T1D patients, and this suggests that CXCL10 may be a candidate for a predictive marker of T1D. Blocking the CXCL10/CXCR3 axis in newly onset of diabetes seems to be a possible approach for the therapy of T1D. Attempts have been done in modulating or blocking CXCR5, CXCR6 and CXCR7 chemokine receptors in experimental settings of T1D. Further studies are needed to investigate interactions between chemokines and cytokines in the pathogenesis and therapy of T1D.
\end{abstract}

Running Title: CXCR3, CXCR5, CXCR6, and CXCR7 in T1D.

Keywords: CXCL10, CXCR3, CXCR5, CXCR6, CXCR7, T1D.

\footnotetext{
* Corresponding Author:

Alessandro Antonelli, Prof

Department of Clinical and Experimental Medicine

University of Pisa,

Via Savi, 10, I-56126, Pisa, Italy

Phone: +39-050-992318

Fax: +39-050-553235

e-mail: alessandro.antonelli@med.unipi.it
} 


\section{Introduction}

Diabetes mellitus type 1 (also known as type 1 diabetes, or T1DM; or insulin-dependent diabetes or juvenile diabetes) is a form of diabetes mellitus that results from the autoimmune destruction of the insulin-producing $\beta$-cells in the pancreas. The subsequent lack of insulin leads to increased blood glucose. T1D is an immunologically mediated disease, in which a $\mathrm{T}$ helper (Th)1 immune response leading $\beta$-cells destruction has been shown [1], involving an expansion of autoreactive CD4+ Th cells and CD8+ T cells, autoantibody-producing B cells and activation of the innate immune system [2-4]. As the key immunological mechanisms of the pathogenesis of this disease are still unclear [5, 6], prevention and treatment of T1D are hampered.

Cytokines and chemokines, and their receptors, play an important role in the pathogenesis of T1D and are targets of new therapeutic approaches. Here, we review researches about (C-X-C motif) receptor (CXCR)3, CXCR5, CXCR6 and CXCR7 chemokine receptors in T1D.

\section{CXCR3 and diabetes}

\section{CXCR3 and its chemokines}

CXCR3 receptor is mostly expressed on activated Th1 cells. It is a seven-transmembrane G-protein coupled chemokine receptor, that is determinant in a variety of inflammatory and immunological disorders. (C-X-C motif) ligand (CXCL)10/interferon (IFN)- $\gamma$-induced protein 10 (IP-10),

CXCL9/monokine induced by IFN- $\gamma(\mathrm{MIG})$ and CXCL11/Interferon Inducible T cell $\alpha$ (ITAC) are its ligands. They are expressed by infiltrating leukocytes, endothelial cells, fibroblasts and by epithelial cells following stimulation by IFN- $\gamma$ or Type I IFNs; their expression is synergistically enhanced by tumor necrosis factor (TNF)- $\alpha$ [7-11].

In transplant rejection, a critical role for CXCR3-dependent T cell recruitment is suggested by the strong upregulation of CXCR3 ligand expression and the prevalent expression of CXCR3 on infiltrating $\mathrm{T}$ cells during allograft rejection in human and animal models [12-14]. The potential importance of CXCR3-mediated leukocyte recruitment in the pathology of autoimmune disorders is indicated by the upregulation of CXCR3 ligands and the increased number of CXCR3+ lymphocytes evidenced in chronic inflammatory diseases, as rheumatoid arthritis (RA) [15-18], systemic lupus 
erythematosus (SLE) (19), inflammatory arthritis (19), and multiple sclerosis [20, 21].

CXCL10/IP-10 is the main CXCR3 ligand and its secretion depends on IFN- $\gamma$, that is itself mediated by the interleukin (IL)-12 cytokine family [22]. Upon cytokines stimulation, CXCL10 is secreted by several cell types, including T lymphocytes, monocytes, fibroblasts, thyrocytes, preadipocytes, etc. Therefore, the presence of high levels of CXCL10 in peripheral liquids is a marker of host immune response, particularly Th1 orientated T cells.

Circulating levels of CXCL10 are increasing with age [23]. Furthermore, recent reports have shown that the serum and/or the tissue expressions of CXCL10 are increased in organ specific autoimmune diseases, such as Graves' disease or Graves' ophthalmopathy [24, 25], autoimmune thyroiditis [26, 27], or systemic rheumatological disorders like rheumatoid arthritis [28], systemic lupus erythematosus [29], systemic sclerosis [30, 31], psoriasis or psoriatic arthritis [32, 33], sarcoidosis [34, 35], HCV-related cryoglobulinemia [36, 37], other HCV immune mediated disorders [36-38] and also in liver and thyroid cancers $[39,40]$.

\section{CXCR3 chemokines and diabetes}

The evaluation of circulating levels of CXCL10 in T1D produced different results. Most of the studies observed high levels of serum CXCL10 in children and adults with T1D, expecially in newly diagnosed T1D [41-44]. Only two studies did not find a CXCL10 increase in T1D serum levels [45, 46].

The prototype Th1 chemokine (CXCL10) and the prototype Th2 chemokine (C-C motif) ligand 2 (CCL2)/monocyte chemotactic protein-1 (MCP-1) have been evaluated longitudinally in children with T1D at onset and follow-up [47]. Serum CXCL10 levels were significantly higher in T1D children than in relatives or control children, while CCL2 were similar in patients, relatives and control subjects.

During the follow-up of T1D patients, CXCL10 was significantly reduced, while CCL2 did not change, with respect to baseline. This study first shows that in children with newly diagnosed T1D, raised serum CXCL10 and normal CCL2 concentrations indicate a predominant Th1-driven autoimmune process, which shifts toward Th2 immunity over the first 1-2 years from diagnosis [47]. Studies examining the sources of CXCL10 in T1D showed that it was produced by peripheral blood monocytes and leukocytes $[48,49]$. 
CXCL10 is highly expressed in lymphocytes infiltrating the human islet; and $\beta$-cells, stimulated by cytokines (as IFN- $\gamma$ and TNF- $\alpha$ ), can modulate the autoimmune response releasing CXCL9, CXCL10 and CXCL11. These chemokines induce the migration of Th1 lymphocytes into the islet, that secrete more IFN- $\gamma$ and TNF- $\alpha$, stimulating a further chemokine production by the target cells, that perpetuates the autoimmune cascade. In agreement, CXCL10 has been identified as the prevalent chemokine expressed in vivo in the islet environment of prediabetic animals and T1D patients [50-52].

For the above mentioned reasons, the CXCL10/CXCR3 chemokine system plays a critical role in the autoimmune process and in $\beta$-cell destruction in T1D.

\section{Effect of blocking CXCL10 in T1D}

The effect of CXCL10 neutralization using a T1D model initiated by developmentally regulated presentation of $\beta$-cell antigens has been evaluated. The occurrence of diabetes was suppressed by CXCL10 neutralization, after the administration with cyclophosphamide in non-obese diabetic (NOD) mice, even if CXCL10 neutralization did not significantly inhibit insulitis and did not influence trafficking of effector T cells into the islets. As CXCL10 and CXCR3 were coexpressed on insulinproducing cells, CXCL10 was considered to affect mature and premature $\beta$-cells in an autocrine and/or paracrine manner. In fact, CXCL10 neutralization increased the proliferation of $\beta$-cells and $\beta$-cell mass, without inhibiting insulitis. For these reasons, it was suggested that CXCL10 neutralization could be considered a new therapeutic target for $\beta$-cell survival, during the early stage of T1D and also after islet transplantation [53].

Another paper evaluated the effect of CXCL10 neutralization in a "spontaneous diabetes" model of NOD mice, through CXCL10 DNA vaccination (pCAGGS-CXCL10), that induced the production of anti-CXCL10 antibodies (Ab) in vivo and suppressed the incidence of spontaneous diabetes, in young NOD mice, without inhibiting insulitis or altering the immunological response. pCAGGS-CXCL10 treatment increased the proliferation of pancreatic $\beta$-cells, causing an increase of $\beta$-cell mass in this spontaneous diabetes model as well. For these reasons, it has been suggested that CXCL10 neutralization could be useful for maintaining $\beta$-cell mass at any stage of autoimmune diabetes [54].

\section{CXCR5 and diabetes}


CXCR5, also known as Burkitt lymphoma receptor 1 (BLR1), belongs to the CXC chemokine receptor family, and it is a G protein-coupled seven transmembrane receptor for chemokine CXCL13, encoded by the CXCR5 gene [55].

The CXCR5 gene is specifically expressed in lymphatic tissues, such as follicles in lymph nodes as well as in spleen; it plays an essential role in B cell migration $[56,57]$. CXCL13, also known as B lymphocyte chemoattractant (BLC), is selectively chemotactic for B cells belonging to both the B-1 and B-2 subsets. CXCL13 and its receptor CXCR5 control the organization of B cells within follicles of lymphoid tissues [58] and is expressed highly in liver, spleen, lymph nodes. In T lymphocytes, CXCL13 expression is thought to reflect a germinal center origin of the T cell, particularly a subset of T cells called Follicular B Helper T cells (or Tfh cells) [58].

Lymphocytes that invade nonlymphoid tissues often organize into follicle-like structures known as tertiary lymphoid organs (TLOs). These structures resemble those found in spleen or lymph nodes, but their function is unknown. TLOs are recognized in the NOD mouse model of T1D. In some cases, TLOs have been associated with the B lymphocyte chemoattractant, in fact CXCL13 is present in inflamed islets of NOD mice [59].

Ab blockade of this chemokine unraveled B lymphocyte organization in islet TLOs, without reducing their proportion in the islets. However, loss of B lymphocyte organization in islets did not provide disease protection against T1D [60]. Tfh cells exert an important role in autoimmune diseases; to investigate the role of Tfh cells in patients with T1D and the effect of anti-CD20 monoclonal antibody (rituximab) on Tfh cells in T1D patients, 54 patients with T1D and 37 healthy controls were enrolled, and 20 T1D were treated with rituximab. Increased frequencies of circulating Tfh cells together with enhanced expression of IL-21 were detected in T1D patients. After rituximab therapy, the frequencies of circulating Tfh cells and serum protein-tyrosine-phosphatase-2 autoantibody (IA2A) were decreased, together with the levels of IL-21, IL-6 and Bcl-6. Furthermore, $\beta$-cell function in 10 of 20 patients was improved. These data suggest that Tfh cells may participate in the T1D-related immune responses, and in the disease progression [61].

\section{CXCR6 and diabetes}

CXCL16 is a small CXC chemokine, composed of a CXC chemokine domain, a transmembrane domain and a cytoplasmic tail containing a potential tyrosine phosphorylation [62]. CXCL16 is 
produced by dendritic cells found in the T cell zones of lymphoid organs, and by cells found in the spleen [62]. Cells that bind and migrate in response to CXCL16 include several subsets of T cells, and natural killer T cells [62]. CXCL16 interacts with the chemokine receptor CXCR6, also known as Bonzo $[62,63]$. Expression of CXCL16 is induced by the inflammatory cytokines IFN- $\gamma$ and TNF- $\alpha$ $[63,64]$.

Mesenchymal stem cells (BM-MSCs) are stromal cells with the ability to proliferate and differentiate into many tissues. A minority of BM-MSCs (2\% to $25 \%)$ expressed a restricted set of chemokine receptors [CXCR4, chemokine (C-X3-C motif) receptor 1 (CX3CR1), CXCR6, (C-C motif) receptor (CCR) 1, CCR7] and, accordingly, showed appreciable chemotactic migration in response to the chemokines CXCL12, chemokine (C-X3-C motif) ligand 1 (CX3CL1), CXCL16, CCL3, and CCL19. A population of bona fide MSCs that also expressed CXCR4, CXCR6, CCR1, and CCR7 could be isolated from normal adult human pancreas [65]. Enteroviruses, particularly Coxsackie virus B4 (CVB4), are considered to be involved in the pathogenesis of T1D [66]. A study compared the characteristics of T cell immune response to CVB4 in children with T1D and healthy children with and without human leukocyte antigen (HLA) risk-associated haplotypes (HLA-DR3-DQ2 or HLA-DR4DQ8) for T1D. Peripheral blood mononuclear cells (PBMCs) showed, in children with T1D, a decreased percentage of T cells expressed CCR2, CXCR6, IL-18R, and IL-12Rß2-chain after in vitro stimulation with CVB4 in comparison with healthy children. Moreover, children with T1D had decreased IFN- $\gamma$ secretion in CVB4-stimulated PBMCs, suggesting an impaired type 1 immune response against CVB4. This may lead to a delayed clearance of the virus and, at least partly, explain why children with T1D may be more prone to CVB4 infections and related complications, such as $\beta$ cell damage [67].

Since chemokines and chemokine receptors are key players in the migration of pathogenic T cells into the islets of NOD mice developing T1D, it was studied the expression of Th1- and Th2-associated chemokine receptors, and the two isoforms of CD45 leucocyte antigen on CD4(+) and CD8(+) lymphocytes from T1D and healthy children. It has been shown an increased expression of CCR7 and CD45RA and reduced CD45RO on CD8(+) cells among recent-onset T1D patients. The percentages of CD4(+) cells expressing CXCR3, CXCR6 and CCR5, and the secretion of CXCL10/IP-10, MCP-1, macrophage inflammatory protein (MIP)-1 $\alpha$ and MIP-1 $\beta$ was lower among diabetics. The Authors suggest that low expression of Th1-associated receptors and secretion of chemokines, together with an 
increased amount of CD8(+) cells expressing CD45RA and CCR7 in T1D patients therefore might represent suboptimal Th function in T1D, leading to impaired T cytotoxic responses [68]. A prior exposure to Complete Freund's Adjuvant (CFA) suppresses the severity of experimental autoimmune encephalomyelitis (EAE) and spontaneous diabetes in rodents. It has been shown that CFA adjuvant immunotherapy of EAE requires IFN- $\gamma$, which suppresses development of the Th17 response, and diverts autoreactive T cells away from the central nervous system toward immature myeloid cells expressing CXCL10 and CXCL16 in the lymph nodes, suggesting a similar mechanism in T1D [69].

\section{CXCR7 and diabetes}

CXCR7 belongs to G protein-coupled receptor family, and it is a chemokine receptor able to bind the chemokines CXCL12/stromal cell-derived factor-1 (SDF-1) and CXCL11 [70, 71].

Genome-wide association studies (GWAS) have heralded a new era in susceptibility locus discovery in complex diseases. For T1D, $>40$ susceptibility loci have been discovered. A study integrated T1D GWAS data with protein-protein interactions to construct biological networks of relevance for disease. A total of 17 networks were identified. To substantiate these networks, it was performed expressional profiling in human pancreatic islets exposed to proinflammatory cytokines. Three networks were significantly enriched for cytokine-regulated genes and, thus, likely to play an important role for T1D in pancreatic islets. Eight of the regulated genes (CD83, IFNGR1, IL-17RD, TRAF3IP2, IL-27RA, PLCG2, MYO1B, and CXCR7) in these networks also harbored single nucleotide polymorphisms nominally associated with T1D. Finally, the expression and cytokine regulation of these new candidate genes were confirmed in insulin-secreting INS-1 $\beta$-cells. These results provide novel insight to the mechanisms behind T1D pathogenesis and, thus, may provide the basis for the design of novel treatment strategies [72].

\section{Conclusion}

The above mentioned studies have suggested that CXCR3, CXCR5, CXCR6 and CXCR7 chemokine receptors play a crtical role in the autoimmune process and in $\beta$-cell destruction in TID. In particular serum CXCL10 is high in T1D patients, and this suggests that CXCL10 may be a candidate for a predictive marker of T1D. Blocking the CXCL10/CXCR3 axis in newly onset of diabetes seems to be a 
possible approach for the therapy of T1D. Attempts have been done in modulating or blocking CXCR5,

CXCR6 and CXCR7 chemokine receptors in experimental settings of T1D.

Further studies are needed to investigate interactions between chemokines and cytokines in the pathogenesis and therapy of T1D. 
Conflict of Interest: The authors have nothing to declare.

Funding source: The authors have nothing to declare. 


\section{Acknowledgements:}

\section{Authorship :}

P. Fallahi, A. Antonelli, S.M. Ferrari made substantial contribution in the design of the study, in the acquisition and interpretation of data, and wrote the paper. A. Corrado, A. Di Domenicantonio and G. Frenzilli made contribution in the acquisition of data and their analysis. 


\section{References:}

[1] Lo J, Clare-Salzler MJ. Dendritic cell subsets and type I diabetes: focus upon DC-based therapy. Autoimmun Rev 2006; 5: 419-23.

[2] Bluestone JA, Herold K, Eisenbarth G. Genetics, pathogenesis and clinical interventions in type 1 diabetes. Nature 2010; 464: 1293-300.

[3] Antonelli A, Baj G, Marchetti P, et al. Human anti-CD38 autoantibodies raise intracellular calcium and stimulate insulin release in human pancreatic islets. Diabetes 2001; 50: 985-91.

[4] Chatzigeorgiou A, Harokopos V, Mylona-Karagianni C, Tsouvalas E, Aidinis V, Kamper EF. The pattern of inflammatory/anti-inflammatory cytokines and chemokines in type 1 diabetic patients over time. Ann Med 2010; 42: 426-38.

[5] Barbeau WE. What is the key environmental trigger in type 1 diabetes--is it viruses, or wheat gluten, or both? Autoimmun Rev 2012; 12: 295-9.

[6] Askenasy EM, Askenasy N. Is autoimmune diabetes caused by aberrant immune activity or defective suppression of physiological self-reactivity? Autoimmun Rev 2013; 12: 633-7.

[7] Cole KE, Strick CA, Paradis TJ, et al. Interferon-inducible T cell alpha chemoattractant (I-TAC): a novel non-ELR CXC chemokine with potent activity on activated $\mathrm{T}$ cells through selective high affinity binding to CXCR3. J Exp Med 1998; 187: 2009-21.

[8] Loetscher M, Gerber B, Loetscher P, et al. Chemokine receptor specific for IP10 and mig: structure, function, and expression in activated T-lymphocytes. J Exp Med 1996; 184: 963-9.

[9] Murphy PM, Baggiolini M, Charo IF, et al. International union of pharmacology. XXII.

Nomenclature for chemokine receptors. Pharmacol Rev 2000; 52: 145-76.

[10] Farber JM. Mig and IP-10: CXC chemokines that target lymphocytes. J

Leukoc Biol 1997; 61: 246-57.

[11] Loetscher M, Loetscher P, Brass N, Meese E, Moser B. Lymphocyte-specific chemokine receptor CXCR3: regulation, chemokine binding and gene localization. Eur J Immunol 1998; 28: 3696-705.

[12] Melter M, Exeni A, Reinders ME, et al. Expression of the chemokine receptor CXCR3 and its ligand IP-10 during human cardiac allograft rejection. Circulation 2001; 104: 2558-64.

[13] Agostini C, Calabrese F, Rea F, et al. Cxcr3 and its ligand CXCL10 are expressed by inflammatory cells infiltrating lung allografts and mediate chemotaxis of T cells at sites of rejection. Am J Pathol 2001; 158: 1703-11. 
[14] Hancock WW, Wang L, Ye Q, Han R, Lee I. Chemokines and their receptors as markers of allograft rejection and targets for immunosuppression. Curr Opin Immunol 2003; 15: 479-86.

[15] Patel DD, Zachariah JP, Whichard LP. CXCR3 and CCR5 ligands in rheumatoid arthritis synovium. Clin Immunol 2001; 98: 39-45.

[16] Qin S, Rottman JB, Myers P, et al. The chemokine receptors CXCR3 and CCR5 mark subsets of T cells associated with certain inflammatory reactions. J Clin Invest 1998; 101: 746-54.

[17] Katschke KJ Jr, Rottman JB, Ruth JH, et al. Differential expression of chemokine receptors on peripheral blood, synovial fluid, and synovial tissue monocytes/macrophages in rheumatoid arthritis. Arthritis Rheum 2001; 44: 1022-32.

[18] Wedderburn LR, Robinson N, Patel A, Varsani H, Woo P. Selective recruitment of polarized T cells expressing CCR5 and CXCR3 to the inflamed joints of children with juvenile idiopathic arthritis. Arthritis Rheum 2000; 43: 765-74.

[19] Antonelli A, Ferrari SM, Giuggioli D, Ferrannini E, Ferri C, Fallahi P. Chemokine (C-X-C motif) ligand (CXCL)10 in autoimmune diseases. Autoimmun Rev 2014; 13: 272-80.

[20] Sørensen TL, Tani M, Jensen J, et al. Expression of specific chemokines and chemokine receptors in the central nervous system of multiple sclerosis patients. J Clin Invest 1999; 103: 807-15.

[21] Balashov KE, Rottman JB, Weiner HL, Hancock WW. CCR5(+) and CXCR3(+) T cells are increased in multiple sclerosis and their ligands MIP-1alpha and IP-10 are expressed in demyelinating brain lesions. Proc Natl Acad Sci USA 1999; 96: 6873-8.

[22] Engel MA, Neurath MF. Anticancer properties of the IL-12 family--focus on colorectal cancer. Curr Med Chem 2010; 3303-8.

[23] Antonelli A, Rotondi M, Fallahi P, et al. Increase of CXC chemokine CXCL10 and CC chemokine CCL2 serum levels in normal ageing. Cytokine 2006; 34: 32-8.

[24] Antonelli A, Ferrari SM, Frascerra S, et al. CXCL9 and CXCL11 chemokines modulation by peroxisome proliferator-activated receptor-alpha agonists secretion in Graves' and normal thyrocytes. J Clin Endocrinol Metab 2010; 95: E413-20.

[25] Antonelli A, Ferrari SM, Fallahi P, et al. Cytokines (interferon- $\gamma$ and tumor necrosis factor- $\alpha$ )induced nuclear factor- $\kappa \mathrm{B}$ activation and chemokine (C-X-C motif) ligand 10 release in Graves disease and ophthalmopathy are modulated by pioglitazone. Metabolism 2011;60:277-83. 
[26] Antonelli A, Ferrari SM, Frascerra S, et al. Circulating chemokine (CXC motif) ligand (CXCL)9 is increased in aggressive chronic autoimmune thyroiditis, in association with CXCL10. Cytokine 2011; 55: 288-93.

[27] Antonelli A, Ferrari SM, Frascerra S, et al. Increase of circulating CXCL9 and CXCL11 associated with euthyroid or subclinically hypothyroid autoimmune thyroiditis. J Clin Endocrinol Metab 2011; 96: 1859-63.

[28] Lee EY, Lee ZH, Song YW. The interaction between CXCL10 and cytokines in chronic inflammatory arthritis. Autoimmun Rev 2013; 12: 554-7.

[29] Lacotte S, Brun S, Muller S, Dumortier H. CXCR3, inflammation, and autoimmune diseases. Ann N Y Acad Sci 2009; 1173: 310-7.

[30] Antonelli A, Ferri C, Fallahi P, et al. Clinical and subclinical autoimmune thyroid disorders in systemic sclerosis. Eur J Endocrinol 2007; 156: 431-7.

[31] Antonelli A, Ferri C, Fallahi P, et al. Th1 and Th2 chemokine serum levels in systemic sclerosis in the presence or absence of autoimmune thyroiditis. J Rheumatol 2008; 35: 1809-11.

[32] Antonelli A, Delle Sedie A, Fallahi P, et al. High prevalence of thyroid autoimmunity and hypothyroidism in patients with psoriatic arthritis. J Rheumatol 2006; 33: 2026-8.

[33] Antonelli A, Fallahi P, Delle Sedie A, et al. High values of alpha (CXCL10) and beta (CCL2) circulating chemokines in patients with psoriatic arthritis, in presence or absence of autoimmune thyroiditis. Autoimmunity 2008; 41: 537-42.

[34] Su R, Nguyen ML, Agarwal MR, et al. Interferon-inducible chemokines reflect severity and progression in sarcoidosis. Respir Res 2013; 14: 121.

[35] Antonelli A, Fazzi P, Fallahi P, Ferrari SM, Ferrannini E. Prevalence of hypothyroidism and Graves disease in sarcoidosis. Chest 2006; 130: 526-32.

[36] Antonelli A, Ferri C, Fallahi P, et al. High values of CXCL10 serum levels in mixed cryoglobulinemia associated with hepatitis C infection. Am J Gastroenterol 2008; 103: 2488-94. [37] Antonelli A, Ferri C, Fallahi P, et al. Alpha-chemokine CXCL10 and beta-chemokine CCL2 serum levels in patients with hepatitis C-associated cryoglobulinemia in the presence or absence of autoimmune thyroiditis. Metabolism. 2008; 57: 1270-7. 
[38] Antonelli A, Ferri C, Fallahi P, et al. High values of CXCL10 serum levels in patients with hepatitis $\mathrm{C}$ associated mixed cryoglobulinemia in presence or absence of autoimmune thyroiditis. Cytokine 2008; 42: 137-43.

[39] Brownell J, Polyak SJ. Molecular pathways: hepatitis C virus, CXCL10, and the inflammatory road to liver cancer. Clin Cancer Res 2013; 19: 1347-52.

[40] Antonelli A, Ferrari SM, Fallahi P, et al. Dysregulation of secretion of CXC alpha-chemokine CXCL10 in papillary thyroid cancer: modulation by peroxisome proliferator-activated receptor-gamma agonists. Endocr Relat Cancer 2009; 16: 1299-311.

[41] Shimada A, Morimoto J, Kodama K, et al. Elevated serum IP-10 levels observed in type 1 diabetes. Diabetes Care 2001; 24: 510-5.

[42] Gabbay MA, Sato MN, Duarte AJ, Dib SA. Serum titres of anti-glutamic acid decarboxylase-65 and anti-IA-2 autoantibodies are associated with different immunoregulatory milieu in newly diagnosed type 1 diabetes patients. Clin Exp Immunol 2012; 168: 60-7.

[43] Nakagawa Y, Shimada A, Oikawa Y, et al. Two cases of "fulminant" type 1 diabetes suggesting involvement of autoimmunity. Ann N Y Acad Sci 2003; 1005: 359-61.

[44] Nicoletti F, Conget I, Di Mauro M, et al. Serum concentrations of the interferon-gamma-inducible chemokine IP-10/CXCL10 are augmented in both newly diagnosed Type I diabetes mellitus patients and subjects at risk of developing the disease, Diabetologia 2002; 45: 1107-10.

[45] Rotondi M, Romagnani P, Brozzetti A, Santeusanio F, Serio M, Falorni A. -to: Nicoletti F, Conget L, Di Mauro M et al. (2002) Serum concentrations of the interferon-alpha-inducible chemokine IP10/CXCL10 are augmented in both newly-diagnosed Type I diabetes mellitus patients and subjects at risk of developing the disease. Diabetologia 45:1107-1110. Diabetologia 2003; 46: 1020-1.

[46] Hanifi-Moghaddam P, Kappler S, Seissler J, et al. Altered chemokine levels in individuals at risk of Type 1 diabetes mellitus. Diabet Med 2006; 23: 156-63.

[47] Antonelli A, Fallahi P, Ferrari SM, et al. Serum Th1 (CXCL10) and Th2 (CCL2) chemokine levels in children with newly diagnosed Type 1 diabetes: a longitudinal study. Diabet Med 2008; 25 : $1349-53$.

[48] Shanmugam N, Ransohoff RM, Natarajan R. Interferon-gamma-inducible protein (IP)-10 mRNA stabilized by RNA-binding proteins in monocytes treated with S100b. J Biol Chem 2006; 281: 3121221. 
[49] Rosa JS, Mitsuhashi M, Oliver SR, et al. Ex vivo TCR-induced leukocyte gene expression of inflammatory mediators is increased in type 1 diabetic patients but not in overweight children. Diabetes Metab Res Rev 2010; 26: 33-9.

[50] Roep BO, Kleijwegt FS, van Halteren AG, et al. Islet inflammation and CXCL10 in recent-onset type 1 diabetes. Clin Exp Immunol 2010; 159: 338-43.

[51] Frigerio S, Junt T, Lu B, et al. Beta cells are responsible for CXCR3-mediated T-cell infiltration in insulitis. Nat Med 2002; 8: 1414-20.

[52] Uno S, Imagawa A, Saisho K, et al. Expression of chemokines, CXC chemokine ligand 10 (CXCL10) and CXCR3 in the inflamed islets of patients with recent-onset autoimmune type 1 diabetes. Endocr J 2010; 57: 991-6.

[53] Morimoto J, Yoneyama H, Shimada A, et al. CXC chemokine ligand 10 neutralization suppresses the occurrence of diabetes in nonobese diabetic mice through enhanced beta cell proliferation without affecting insulitis. J Immunol 2004; 173: 7017-24.

[54] Shigihara T, Shimada A, Oikawa Y, et al. CXCL10 DNA vaccination prevents spontaneous diabetes through enhanced beta cell proliferation in NOD mice. J Immunol 2005; 175: 8401-8.

[55] Gunn MD, Ngo VN, Ansel KM, Ekland EH, Cyster JG, Williams LT. A B-cell-homing chemokine made in lymphoid follicles activates Burkitt's lymphoma receptor-1. Nature 1998; 391: 799-803.

[56] Dobner T, Wolf I, Emrich T, Lipp M. Differentiation-specific expression of a novel G proteincoupled receptor from Burkitt's lymphoma. Eur J Immunol 1992; 22: 2795-9.

[57] Förster R, Mattis AE, Kremmer E, Wolf E, Brem G, Lipp M. A putative chemokine receptor, BLR1, directs B cell migration to defined lymphoid organs and specific anatomic compartments of the spleen. Cell 1996; 87: 1037-47.

[58] Corsiero E, Bombardieri M, Manzo A, Bugatti S, Uguccioni M, Pitzalis C. Role of lymphoid chemokines in the development of functional ectopic lymphoid structures in rheumatic autoimmune diseases. Immunol Lett 2012; 145: 62-7.

[59] Astorri E, Bombardieri M, Gabba S, Peakman M, Pozzilli P, Pitzalis C. Evolution of ectopic lymphoid neogenesis and in situ autoantibody production in autoimmune nonobese diabetic mice: cellular and molecular characterization of tertiary lymphoid structures in pancreatic islets. J Immunol 2010; 185: 3359-68. 
[60] Henry RA, Kendall PL.CXCL13 blockade disrupts B lymphocyte organization in tertiary lymphoid structures without altering B cell receptor bias or preventing diabetes in nonobese diabetic mice. J Immunol 2010; 185: 1460-5.

[61] Xu X, Shi Y, Cai Y, et al. Inhibition of increased circulating Tfh cell by anti-CD20 monoclonal antibody in patients with type 1 diabetes. PLoS One 2013; 8: e79858.

[62] Abel S, Hundhausen C, Mentlein R, et al. The transmembrane CXC-chemokine ligand 16 is induced by IFN-gamma and TNF-alpha and shed by the activity of the disintegrin-like metalloproteinase ADAM10. J Immunol 2004; 172: 6362-72.

[63] Wilbanks A, Zondlo S, Murphy K, et al. Expression cloning of the STRL33/BONZO/TYMSTRligand reveals elements of CC, CXC, and CX3C chemokines. J Immunol 2001; 166: 5145-54.

[64] Matloubian M, David A, Engel S, Ryan JE, Cyster JC. A transmembrane CXC chemokine is a ligand for HIV-coreceptor Bonzo. Nat Immunol 2000; 1: 298-304.

[65] Sordi V, Malosio ML, Marchesi F, et al. Bone marrow mesenchymal stem cells express a restricted set of functionally active chemokine receptors capable of promoting migration to pancreatic islets. Blood 2005; 106: 419-27.

[66] Antonelli A, Ferrari SM, Corrado A, Ferrannini E, Fallahi P. CXCR3, CXCL10 and type 1 diabetes. Cytokine Growth Factor Rev 2014; 25: 57-65.

[67] Skarsvik S, Puranen J, Honkanen J, et al. Decreased in vitro type 1 immune response against coxsackie virus B4 in children with type 1 diabetes. Diabetes 2006; 55: 996-1003.

[68] Hedman M, Faresjö M, Axelsson S, Ludvigsson J, Casas R. Impaired CD4 and CD8 T cell phenotype and reduced chemokine secretion in recent-onset type 1 diabetic children. Clin Exp Immunol 2008; 153: 360-8.

[69] O'Connor RA, Li X, Blumerman S, Anderton SM, Noelle RJ, Dalton DK. Adjuvant immunotherapy of experimental autoimmune encephalomyelitis: immature myeloid cells expressing CXCL10 and CXCL16 attract CXCR3+CXCR6+ and myelin-specific T cells to the draining lymph nodes rather than the central nervous system. J Immunol 2012; 188: 2093-101.

[70] Balabanian K, Lagane B, Infantino S, et al. The chemokine SDF-1/CXCL12 binds to and signals through the orphan receptor RDC1 in T lymphocytes. J Biol Chem 2005; 280: 35760-6. 
[71] Rajagopal S, Kim J, Ahn S, et al. Beta-arrestin- but not G protein-mediated signaling by the "decoy" receptor CXCR7. Proc Natl Acad Sci U S A. 2010; 107: 628-32.

[72] Bergholdt R, Brorsson C, Palleja A, et al. Identification of novel type 1 diabetes candidate genes by integrating genome-wide association data, protein-protein interactions, and human pancreatic islet gene expression. Diabetes 2012; 61: 954-62. 